\title{
COVID-19 vaccine hesitancy January-May 2021 among 18-64 year old US adults by employment and occupation
}

\author{
Wendy C. King ${ }^{a}$, Max Rubinstein ${ }^{b}$, Alex Reinhart ${ }^{c}$, Robin Mejia ${ }^{c, *}$ \\ a Department of Epidemiology, Graduate School of Public Health University of Pittsburgh, Pittsburgh, PA, USA \\ ${ }^{\mathrm{b}}$ Heinz College and Department of Statistics \& Data Science, Carnegie Mellon University, Pittsburgh, PA, USA \\ ${ }^{\mathrm{c}}$ Department of Statistics \& Data Science, Carnegie Mellon University, Pittsburgh, PA, USA
}

\section{A R T I C L E I N F O}

\section{Keywords:}

SARS-CoV-2

United States

Workforce

Profession

Vaccination

\begin{abstract}
A B S T R A C T
COVID-19 vaccine hesitancy threatens pandemic control efforts. We evaluated vaccine hesitancy in the US by employment status and occupation category during the COVID-19 vaccine rollout. US adults 18-64 years completed an online COVID-19 survey 3,179,174 times from January 6-May 19, 2021. Data was aggregated by month. Survey weights matched the sample to the US population age, gender, and state profile. Weighted percentages and 95\% confidence intervals (CI) were calculated. Changes in vaccine hesitancy from January-May varied widely by employment status (e.g., $-7.8 \%$ [95\%CI, $-8.2--7.5]$ among those working outside the home, a $26.6 \%$ decrease; -13.3\% [95\%CI, -13.7 - - 13.0] among those not working for pay, a 44.9\% decrease), and occupation category (e.g., $-15.9 \%$ [95\%CI, $-17.7--14.2]$ in production, a 39.3\% decrease; $-1.4 \%$ [95\% $\mathrm{CI},-3.8--1.0]$ in construction/extraction, a 3.0\% decrease). April 20-May 19, 2021, vaccine hesitancy ranged from 7.3\% (95\%CI, $6.7-7.8$ ) in computer/mathematical professions to $45.2 \%$ (95\%CI, 43.2-46.8) in construction/extraction. Hesitancy was 9.0\% (95\%CI, 8.6-9.3) among educators and 14.5\% (95\%CI, 14.0-15.0) among healthcare practitioners/technicians. While the prevalence of reasons for hesitancy differed by occupation, over half of employed hesitant participants reported concern about side effects (51.7\%) and not trusting COVID-19 vaccines (51.3\%), whereas only 15.0\% didn't like vaccines in general. Over a third didn't believe they needed the vaccine, didn't trust the government, and/or were waiting to see if it was safe. In this massive national survey of adults 18-64 years, vaccine hesitancy varied widely by occupation. Reasons for hesitancy indicate messaging about safety and addressing trust are paramount.
\end{abstract}

\section{Introduction}

The development of highly efficacious COVID-19 vaccines in less than one year is a major medical accomplishment of the last century. However, vaccine hesitancy (i.e., a refusal or reluctance to be vaccinated) has slowed projected uptake (Razai et al., 2021) and remains a barrier COVID-19 pandemic control (Schaffer DeRoo et al., 2020). A longitudinal study of US adults that collected data through the approval and launch of three COVID-19 vaccines reported a decrease in COVID-19 vaccine hesitancy from 46.0\% in October 2020 to 35.2\% in March 2021 (Daly et al., 2021). Still, a greater reduction in vaccine hesitancy is needed to meet uptake goals of 70\%-90\% (Razai et al., 2021).

Adults $\geq 60$ years had a larger decrease in COVID-19 vaccine hesitancy versus younger adults October 2020-March 2021 (Daly et al.,
2021), and, consistent with previous reports (Daly et al., 2021; Khubchandani et al., 2021; Malik et al., 2020), had lower hesitancy at a given time point compared to younger adults. While younger versus older adults are less likely to be hospitalized or die from COVID-19 (Rosenthal et al., 2020), vaccine hesitancy among working-age adults may contribute to workplace outbreaks and spread of infection between workers and customers, healthcare workers and patients, and educators and students, all serious public health threats (Althouse et al., 2020; Gold et al., 2021).

Age, sex, gender, race/ethnicity, education level, and living in an urban versus rural county are known correlates of COVID-19 vaccine hesitancy (Daly et al., 2021; Khubchandani et al., 2021; Malik et al., 2020; Szilagyi et al., 2021; Pew Research Center, 2021). However, very few studies have evaluated COVID-19 vaccine hesitancy by employment

\footnotetext{
* Corresponding author at: Carnegie Mellon University, 5000 Forbes Avenue, Pittsburgh, PA 15213, USA.

E-mail addresses: kingw@edc.pitt.edu (W.C. King), mrubinst@andrew.cmu.edu (M. Rubinstein), areinhar@stat.cmu.edu (A. Reinhart), rmejia@andrew.cmu.edu (R. Mejia).
} 
status; those that did had small samples and were conducted in June 2020 about a then-future vaccine (Khubchandani et al., 2021; Malik et al., 2020), and we know of none comparing COVID-19 vaccine hesitancy by occupation. Elucidating the prevalence of vaccine hesitancy in the US workforce, and in particular, by occupation, is important for understanding risk of transmission and outbreaks in various job settings. Further, understanding why individuals are hesitant and if reasons vary by occupation is important for developing effective campaigns to increase vaccination uptake.

Among a massive sample of working-age (18-64 year old) US adults, we report COVID-19 vaccine hesitancy by month, January 6 through May 19, 2021, and evaluate time trends by employment status and occupation category. For the last 30 days, we report COVID-19 vaccination history and prevalence of COVID-19 vaccine hesitancy by occupation category, and the relative association between occupation category with hesitancy, with and without adjustment for demographics. Given healthcare workers and educators pose transmission risk to vulnerable populations (i.e., to patients and children $<12$ years, who are not yet eligible for vaccination, respectively), we also evaluate hesitancy by profession within each of these occupations. Finally, we identify the most common reasons for COVID-19 vaccine hesitancy among the workforce and by occupation category.

\section{Materials and methods}

\subsection{Sampling and weighting}

Since April 2020, the Delphi group at Carnegie Mellon University (CMU) has been conducting an ongoing national survey, COVID-19 Trends and Impact Survey (Salomon et al., 2021), in collaboration with the Facebook Data for Good group. Each month the survey is offered to a random sample, stratified by geographic region, of $\approx 100$ million US residents from the Facebook Active User Base who use one of the supported languages (English [American and British], Spanish [Spain and Latin American], French, Brazilian Portuguese, Vietnamese, and simplified Chinese). The offer to participate is shown with a link to the survey at the top of users' Facebook News Feed to yield $\approx 1.1$ million responders per month, which allows for evaluation of local trends. When individuals click through the link, an anonymized unique identifier is generated. CMU returns the unique IDs to Facebook, which creates weights that account for the sampling design and non-response; these weights are then post-stratified to match the US general population by age, gender, and state (Barkay et al., 2020). This design safeguards respondent privacy by ensuring that researchers at CMU do not receive an identifying information about respondents and Facebook does not see survey microdata. The CMU Institutional Review Board approved the survey protocol and instrument (STUDY2020_00000162).

\subsection{Study sample}

Facebook users may be offered the survey from once a month to once every six months, depending on their geographic strata. To show trends over time in vaccine hesitancy, we used data from January 6 to May 19, 2021 (a period in which the same version of the vaccine uptake and intent questions were offered to all potential respondents) aggregated by month. While it is possible there are repeat respondents across months, respondents cannot be linked longitudinally, so data was treated as repeat cross-sectional surveys. Only data from the last 30 days (April 20May 19) was used in the cross-sectional analysis of vaccine uptake and hesitancy by occupation category/profession and reasons for hesitancy, avoiding repeat respondents and focusing on the most current data.

April 20-May 19, 2021, 104,760,491 Facebook users were offered the survey, of whom 904,022 completed at least two survey questions. Respondents were excluded if they were 65 or older $(n=224,197)$, did not report their age $(n=153,665)$ or did not answer the vaccine acceptance question $(n=351$ ), leaving 525,809 participants. Applying the same criteria, the January-May monthly samples for time trends had 791,716 ; 710,529; 732,308; 631,621; and 313,000 participants, respectively; study flow by months is reported in Supplemental sTable 1.

\subsection{Measures}

The survey questions and response sets utilized in this report and a listing of professions by occupation category, based on the Bureau of Labor Statistics Standard Occupational Codes (Standard Occupational Classification (SOC) System, 2021), are provided in appendix 1 (Supplemental Material). The gender question was developed for this survey; other demographic questions were adapted from existing surveys: race and ethnicity match the 2020 Census definitions (US Census Bureau, 2021), education categories were adapted from the American Community Survey (US Census Bureau, 2021), age categories match the 10-year blocks reported by the ACS (American Community Survey, 2021). Participant's self-reported home zip code was used to determine the urban-rural level of their metropolitan statistical area classification (Centers for Disease Control and Prevention, 2019). Vaccination questions were adapted from CDC-sponsored questions developed for two household panel surveys (Baack et al., 2021) and shared with us prior to launch. The answer set for reasons for vaccine hesitancy, which appears to be a distinct phenomenon from general vaccine hesitancy, was expanded through a review of media reports and brainstorming sessions among survey methodologists.

For this analysis, participants were categorized as vaccine hesitant if they answered that they would "probably not" or "definitely not" choose to get vaccinated if offered a vaccine to prevent COVID-19 today (versus probably or definitely would choose to get vaccinated or were vaccinated), and as strongly hesitant if they answered "definitely not." Already vaccinated individuals were included in the vaccine accepting category to ensure a consistent study population, as access to vaccinations varied by employment category, state, and month in the studied timeframe. Participants were categorized by employment status in the past 4 weeks (employed for pay, work outside the home; employed for pay, work at home; not employed for pay), and if employed, by occupation category and profession.

\subsection{Statistical analysis}

All estimates were generated using survey weights (Barkay et al., 2020). Percentage vaccine hesitant was calculated by month, overall, by employment status, and by occupation category. The difference in hesitancy from January to May was calculated as the May value minus the January value. The percent change was calculated as the difference divided by the January value. Percentages of employment status categories were also calculated by month to understand temporal trends in employment.

Among the final 30-day sample, percentages for worked outside the home, history of COVID-19 vaccination, and vaccine hesitancy (strong and total) were calculated among employed participants, by occupation categories, and by profession among health care practitioners/technicians, healthcare support and educators due their contact with vulnerable populations (i.e., patients, who may be high-risk for poor COVID-19 outcomes, or children, who may not yet be eligible for vaccination). Additionally, risk ratios (RR) for vaccine hesitancy by occupation category were calculated using Poisson regression. Adjusted RR were also calculated controlling for gender, age, race/ethnicity, education level, and urban-rural classification.

Finally, percentages for reasons for hesitancy were calculated among all employed vaccine hesitant participants; among healthcare practitioners/technicians, healthcare support, and educators; among the 5 occupation categories with the highest hesitancy prevalence, and among an additional 5 occupation categories with high-density indoor workspaces or significant client contact. For all parameters, 95\% confidence intervals $(95 \% \mathrm{CI})$ were calculated using robust standard errors 
a

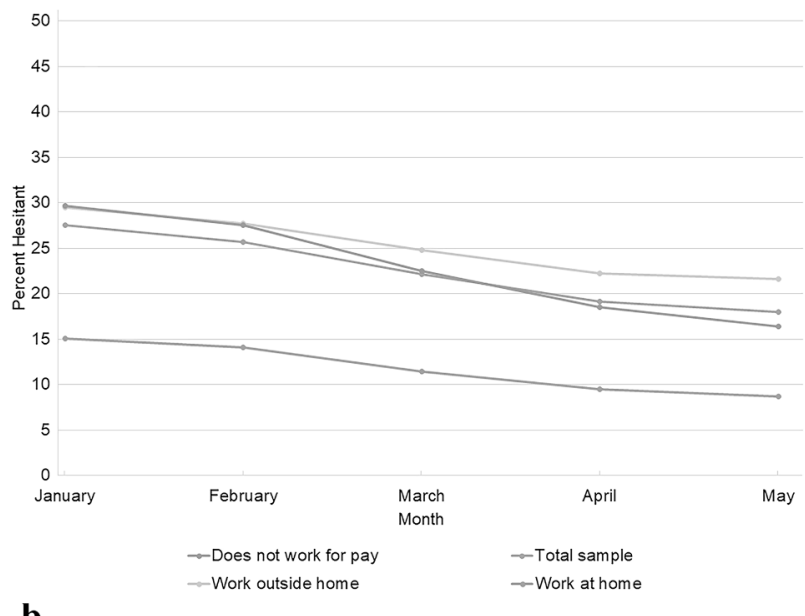

b

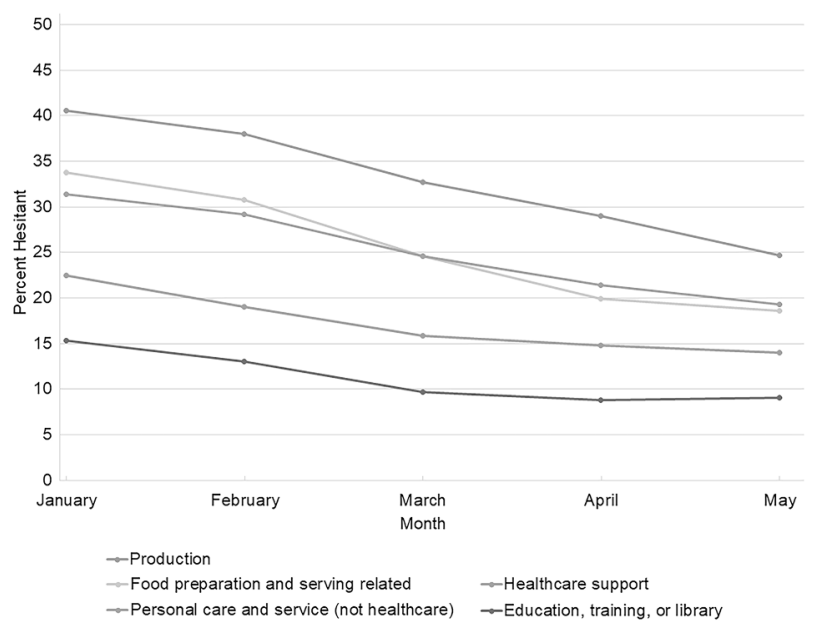

$\mathbf{c}_{50}$

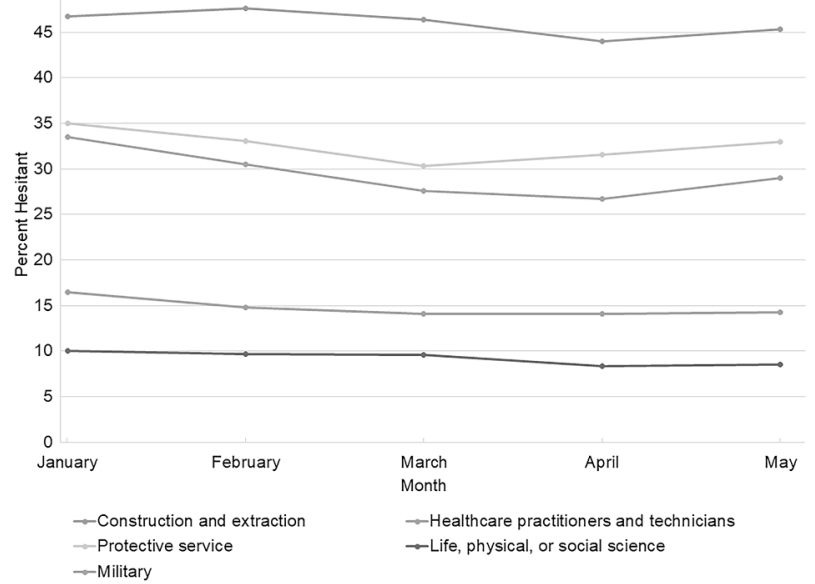

(caption on next column)
Fig. 1. Prevalence of COVID-19 vaccine hesitancy among 18-64 year-old US adults $(\mathrm{N}=3,179,174)$ by employment status (A) and select occupational categories with substantial (B) and little change (C), by month, Jan-May 2021. There was a decrease in COVID-19 vaccine hesitancy prevalence between January and May 2021, among all employment status categories (panel A). There was a smaller change among those who worked outside the home ( $-7.8 \%$; a 26.6 percent decrease) compared to those who did not work for pay (-13.3\%; a 44.9 percent decrease). There was considerable variability in change in prevalence of COVID-19 vaccine hesitancy by occupation category (panels B and C). While most occupations with substantial change in hesitancy had starting points that were relatively high (e.g., production), there were exceptions (e.g., educators) (panel B). Occupations with only small changes in hesitancy from January to May include both those with relatively high starting points (e.g., construction/extraction) and low starting points (e.g., healthcare practitioners/technicians) (panel C).

(Freedman, 2006). Analyses were conducted in R (Version 4.0.2, R Core Team, Vienna, Austria). Code is available in appendix 2 (Supplemental Material).

\section{Results}

\subsection{Participant characteristics}

Final month (April 20-May 19) participants ( $N=525,809)$ had a median age range of $35-44$ years; $45.5 \%$ were male, $52.0 \%$ female, $1.3 \%$ non-binary, and $1.2 \%$ self-described gender; $16.7 \%$ were Hispanic, 68.8\% White, 6.5\% Black, 3.6\% Asian, 0.9\% Native American, 0.3\% Pacific Islander, and 3.4\% Multi-racial; $23.2 \%$ had $\leq$ high school education, $40.7 \%$ had $\geq$ four-year college; $13.4 \%$ lived in a non-core or micropolitan area, $50.4 \%$ lived in a large central or fringe metro area. Two-thirds (66.1\%) worked for pay; half $(50.6 \%)$ worked outside the home. Demographics were similar across all months (data not shown), including employment status. Compared to January, in May: 1.7\% more participants reported working outside the home, while $1.2 \%$ fewer reported working at home, and $0.4 \%$ fewer reported not working for pay (eTable 2).

\subsection{January-May time trends}

As shown in Fig. 1 panel A and reported in eTable2, vaccine hesitancy decreased 9.5 (95\%CI, 9.3-9.7) percentage points, a 34.5\% (95\% CI, 35.2-33.8) decrease, from January (27.4\% [95\%CI, 27.3-27.6]) to May (18.0\% [95\%CI, 17.8-18.1]). There was a smaller relative decrease among those who worked outside the home (7.8 percentage points; a $26.6 \%$ decrease) versus those who worked from home (6.4 percentage points; a $42.4 \%$ decrease) or did not work for pay (13.3 percentage points; a $44.9 \%$ decrease).

Figure 1, panels B and C shows trajectories of COVID-19 vaccine hesitancy January to May for select occupation categories, highlighting that both the prevalence of hesitancy at a given time point and the degree of change in hesitancy prevalence over time varied by occupation category. While most occupations with substantial change in hesitancy had a relatively high hesitancy prevalence in January (e.g., production, food preparation/serving, personal care/service), there were exceptions (e.g., education/training/library and healthcare support) (panel B). Occupations with only small changes in hesitancy from January to May include both those with a relatively high hesitancy prevalence in January (e.g., construction/extraction, protective services, military) and 
Table 1

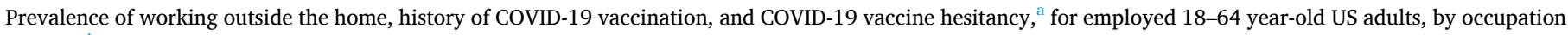
category $^{\mathrm{b}}$, April 20-May 19, 2021. Rate ratios for vaccine hesitancy compared to Educators as the reference are also reported.

\begin{tabular}{|c|c|c|c|c|c|c|c|}
\hline & \multirow[t]{2}{*}{$\mathrm{N}$} & \multirow{2}{*}{$\begin{array}{l}\text { Work outside } \\
\text { home } \\
\%(95 \% \mathrm{CI})\end{array}$} & \multirow{2}{*}{$\begin{array}{l}\text { Vaccinated } \\
\%(95 \% \mathrm{CI})\end{array}$} & \multirow{2}{*}{$\begin{array}{l}\begin{array}{l}\text { Strongly } \\
\text { hesitant }^{\mathrm{a}}\end{array} \\
\%(95 \% \mathrm{CI})\end{array}$} & \multicolumn{3}{|l|}{ Hesitant $^{\mathrm{a}}$} \\
\hline & & & & & $\%(95 \% \mathrm{CI})$ & $\mathrm{RR}(95 \% \mathrm{CI})$ & aRR (95\% CI) \\
\hline Employed & 338,226 & $75.6(75.5,75.8)$ & $\begin{array}{l}75.2 \text { (75.0, } \\
75.4)\end{array}$ & $13.0(12.9,13.2)$ & $\begin{array}{l}19.0(18.8 \\
19.1)\end{array}$ & Not applicable & Not applicable \\
\hline \multicolumn{8}{|l|}{ Occupation } \\
\hline Computer and mathematical & 13,047 & $24.7(23.8,25.5)$ & $\begin{array}{l}89.0 \text { (88.3, } \\
89.7)\end{array}$ & $4.4(3.9,4.8)$ & $7.3(6.7,7.8)$ & $\begin{array}{l}0.81(0.74, \\
0.88)\end{array}$ & $\begin{array}{l}0.90(0.82, \\
0.98)\end{array}$ \\
\hline Life, physical, or social science & 3152 & $66.9(64.9,68.9)$ & $\begin{array}{l}89.1 \text { (87.6, } \\
90.6)\end{array}$ & $5.3(4.3,6.4)$ & $8.3(7.0,9.5)$ & $\begin{array}{l}0.92(0.78, \\
1.07)\end{array}$ & $\begin{array}{l}0.85(0.72, \\
0.99)\end{array}$ \\
\hline Education, training, or library & 32,485 & $78.7(78.2,79.3)$ & $\begin{array}{l}88.7 \text { (88.2, } \\
89.1)\end{array}$ & $5.7(5.4,6.0)$ & $9.0(8.6,9.3)$ & Reference & Reference \\
\hline $\begin{array}{l}\text { Arts, design, entertainment, sports, and } \\
\text { media }\end{array}$ & 10,887 & $53.8(52.6,55.0)$ & $\begin{array}{l}86.3(85.5 \\
87.1)\end{array}$ & $5.6(5.1,6.1)$ & $9.0(8.4,9.7)$ & $\begin{array}{l}1.01(0.92, \\
1.10)\end{array}$ & $\begin{array}{l}0.95(0.87 \\
1.03)\end{array}$ \\
\hline Legal & 4464 & $58.9(57.2,60.6)$ & $\begin{array}{l}87.9 \text { (86.5, } \\
89.2)\end{array}$ & $7.2(6.1,8.3)$ & $9.9(8.6,11.1)$ & $\begin{array}{l}1.10(0.95, \\
1.25)\end{array}$ & $\begin{array}{l}1.07(0.92, \\
1.21)\end{array}$ \\
\hline Business and finance operations & 10,393 & $38.5(37.3,39.6)$ & $\begin{array}{l}83.3 \text { (82.3, } \\
84.2)\end{array}$ & $8.0(7.3,8.7)$ & $\begin{array}{l}12.4(11.5, \\
13.2)\end{array}$ & $\begin{array}{l}1.38(1.27, \\
1.49)\end{array}$ & $\begin{array}{l}1.42(1.31, \\
1.52)\end{array}$ \\
\hline Office and administrative support & 44,859 & $63.3(62.8,63.8)$ & $\begin{array}{l}83.3(82.9, \\
83.8)\end{array}$ & $7.9(7.6,8.2)$ & $\begin{array}{l}12.6(12.2, \\
13.0)\end{array}$ & $\begin{array}{l}1.41(1.33, \\
1.48)\end{array}$ & $\begin{array}{l}1.32(1.25, \\
1.39)\end{array}$ \\
\hline Community and social service ${ }^{c}$ & 14,376 & $80.2(79.5,81.0)$ & $\begin{array}{l}83.1 \text { (82.2, } \\
83.9)\end{array}$ & $8.4(7.8,9.1)$ & $\begin{array}{l}13.4(12.7, \\
14.2)\end{array}$ & $\begin{array}{l}1.50(1.39, \\
1.61)\end{array}$ & $\begin{array}{l}1.42(1.32, \\
1.52)\end{array}$ \\
\hline Management & 13,319 & $56.6(55.6,57.5)$ & $\begin{array}{l}82.8(82.0, \\
83.6)\end{array}$ & $10.0(9.3,10.7)$ & $\begin{array}{l}14.3(13.5, \\
15.1)\end{array}$ & $\begin{array}{l}1.60(1.49, \\
1.71)\end{array}$ & $\begin{array}{l}1.62(1.52, \\
1.73)\end{array}$ \\
\hline Healthcare support & 18,106 & $78.7(78.0,79.4)$ & $\begin{array}{l}81.5 \text { (80.8, } \\
82.3)\end{array}$ & $9.4(8.8,9.9)$ & $\begin{array}{l}14.4(13.7, \\
15.0)\end{array}$ & $\begin{array}{l}1.61(1.50, \\
1.71)\end{array}$ & $\begin{array}{l}1.31(1.23, \\
1.40)\end{array}$ \\
\hline Healthcare practitioners and technicians & 27,080 & $93.9(93.5,94.2)$ & $\begin{array}{l}83.3 \text { (82.8, } \\
83.9)\end{array}$ & $10.5(10.0,10.9)$ & $\begin{array}{l}14.5(14.0, \\
15.0)\end{array}$ & $\begin{array}{l}1.62(1.53, \\
1.71)\end{array}$ & $\begin{array}{l}1.42(1.34, \\
1.50)\end{array}$ \\
\hline Architecture and engineering & 4559 & $67.0(65.4,68.7)$ & $\begin{array}{l}79.9 \text { (78.3, } \\
81.5)\end{array}$ & $11.7(10.3,13.0)$ & $\begin{array}{l}16.5(15.0, \\
18.1)\end{array}$ & $\begin{array}{l}1.85(1.66, \\
2.04)\end{array}$ & $\begin{array}{l}1.84(1.66, \\
2.01)\end{array}$ \\
\hline Food preparation and serving related & 19,334 & $96.2(95.9,96.5)$ & $\begin{array}{l}70.3(69.4 \\
71.1)\end{array}$ & $11.4(10.8,12.0)$ & $\begin{array}{l}19.0(18.3 \\
19.8)\end{array}$ & $\begin{array}{l}2.13(2.00, \\
2.25)\end{array}$ & $\begin{array}{l}1.49(1.41 \\
1.58)\end{array}$ \\
\hline Personal care and service (not healthcare) & 6899 & $82.5(81.5,83.6)$ & $\begin{array}{l}71.9 \text { (70.6, } \\
73.3)\end{array}$ & $12.2(11.3,13.2)$ & $\begin{array}{l}19.7(18.5, \\
20.8)\end{array}$ & $\begin{array}{l}2.20(2.04, \\
2.36)\end{array}$ & $\begin{array}{l}1.71 \text { (1.59, } \\
1.83)\end{array}$ \\
\hline Sales and related & 27,095 & $79.3(78.7,79.9)$ & $\begin{array}{l}70.9(70.2 \\
71.6)\end{array}$ & $14.5(14.0,15.1)$ & $\begin{array}{l}21.8(21.1 \\
22.4)\end{array}$ & $\begin{array}{l}2.43(2.31 \\
2.56)\end{array}$ & $\begin{array}{l}1.94(1.83 \\
2.04)\end{array}$ \\
\hline $\begin{array}{l}\text { Building and grounds cleaning/ } \\
\text { maintenance }\end{array}$ & 6706 & $94.2(93.5,94.9)$ & $\begin{array}{l}64.7(63.2 \\
66.2)\end{array}$ & $15.0(13.9,16.2)$ & $\begin{array}{l}22.9(21.6 \\
24.2)\end{array}$ & $\begin{array}{l}2.56(2.38 \\
2.74)\end{array}$ & $\begin{array}{l}1.95(1.81 \\
2.09)\end{array}$ \\
\hline Production $^{\mathrm{d}}$ & 8409 & $95.5(95.0,96.0)$ & $\begin{array}{l}66.9(65.7 \\
68.1)\end{array}$ & $17.9(16.8,18.9)$ & $\begin{array}{l}25.9(24.8 \\
27.1)\end{array}$ & $\begin{array}{l}2.90(2.71, \\
3.08)\end{array}$ & $\begin{array}{l}2.01(1.88, \\
2.13)\end{array}$ \\
\hline Military & 1517 & $91.6(90.1,93.1)$ & $\begin{array}{l}66.2(62.8 \\
69.6)\end{array}$ & $22.3(19.2,25.4)$ & $\begin{array}{l}28.2(25.0 \\
31.4)\end{array}$ & $\begin{array}{l}3.15(2.76, \\
3.53)\end{array}$ & $\begin{array}{l}2.15(1.89 \\
2.41)\end{array}$ \\
\hline Transportation and material moving & 12,309 & $95.1(94.7,95.6)$ & $\begin{array}{l}62.1(61.1 \\
63.2)\end{array}$ & $21.5(20.6,22.4)$ & $\begin{array}{l}29.6(28.6, \\
30.6)\end{array}$ & $\begin{array}{l}3.31(3.13 \\
3.49)\end{array}$ & $\begin{array}{l}2.43(2.29, \\
2.57)\end{array}$ \\
\hline Protective service & 3916 & $95.4(94.6,96.3)$ & $\begin{array}{l}62.7(60.8 \\
64.5)\end{array}$ & $25.7(24.0,27.4)$ & $\begin{array}{l}32.9(31.1, \\
34.6)\end{array}$ & $\begin{array}{l}3.67(3.41, \\
3.93)\end{array}$ & $\begin{array}{l}2.74(2.55 \\
2.93)\end{array}$ \\
\hline Farming, fishing, and forestry & 2168 & $89.7(88.4,91.1)$ & $\begin{array}{l}53.0(50.4, \\
55.7)\end{array}$ & $30.7(28.2,33.2)$ & $\begin{array}{l}39.1(36.5, \\
41.8)\end{array}$ & $\begin{array}{l}4.37(4.02, \\
4.72)\end{array}$ & $\begin{array}{l}2.66(2.46, \\
2.87)\end{array}$ \\
\hline Installation, maintenance, repair & 8513 & $95.2(94.6,95.7)$ & $\begin{array}{l}52.1(50.8 \\
53.4)\end{array}$ & $28.6(27.4,29.8)$ & $\begin{array}{l}39.3(38.0 \\
40.6)\end{array}$ & $\begin{array}{l}4.39(4.15 \\
4.63)\end{array}$ & $\begin{array}{l}2.97(2.81, \\
3.14)\end{array}$ \\
\hline Construction and extraction $^{e}$ & 6093 & $96.0(95.4,96.6)$ & $\begin{array}{l}46.6(45.1 \\
48.1)\end{array}$ & $34.8(33.2,36.3)$ & $\begin{array}{l}45.2(43.7 \\
46.8)\end{array}$ & $\begin{array}{l}5.05(4.77, \\
5.33)\end{array}$ & $\begin{array}{l}3.29(3.10, \\
3.47)\end{array}$ \\
\hline Any other occupation group & 31,538 & $78.6(78.1,79.1)$ & $\begin{array}{l}65.1(64.4 \\
65.8)\end{array}$ & $18.8(18.2,19.4)$ & $\begin{array}{l}26.4(25.8, \\
27.0)\end{array}$ & $\begin{array}{l}2.95(2.80, \\
3.10)\end{array}$ & $\begin{array}{l}2.17(2.06, \\
2.27)\end{array}$ \\
\hline Employed, occupation not reported & 7002 & $25.2(23.9,26.5$ & $\begin{array}{l}65.8(64.3 \\
67.2)\end{array}$ & $17.3(16.1,18.5)$ & $\begin{array}{l}24.5 \text { (23.2, } \\
25.9)\end{array}$ & $2.74(2.55,2.93$ & $\begin{array}{l}2.00(1.87, \\
2.14)\end{array}$ \\
\hline
\end{tabular}

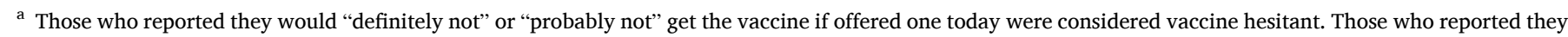
would "definitely not" were considered strongly hesitant.

b Occupation categories were adapted from the Bureau of Labor Statistics Standard Occupational Classification.

c Including counselor, school counselor, mental health worker, social worker, or religious worker.

d Including food processing, meat packing, laundry, and dry cleaning workers.

e Including oil, gas, mining, or quarrying.

a relatively low prevalence (e.g., healthcare practitioners/technicians, life/physical/social science) (panel C). Vaccine hesitancy by month and January to May changes are reported for all occupation categories in eTable3.

\subsection{April 20 - May 19, 2021}

The percentage of respondents who worked outside the home, had received at least one dose of a COVID-19 vaccine, and were COVID-19 vaccine hesitant (strongly and total) during the last 30 days of data collection are reported among employed participants and by occupation category in Table 1. Among employed participants, 75.6\% [95\%CI, 75.5, 75.8]) reported working outside the home. This figure was $>95 \%$ in several occupations (e.g., construction/extraction, protective services). However, more than one-third of respondents reported working from home in business/finance operations, management, legal, arts/design/ 
Table 2

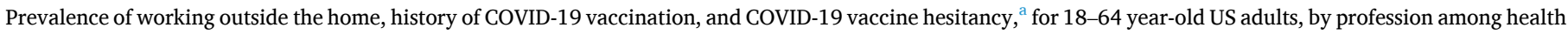

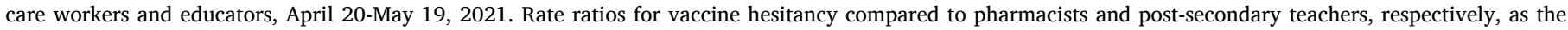
reference are also reported.

\begin{tabular}{|c|c|c|c|c|c|c|c|}
\hline & \multirow[t]{2}{*}{$\mathrm{N}$} & \multirow{2}{*}{$\begin{array}{l}\text { Work outside } \\
\text { home } \\
\%(95 \% \mathrm{CI})\end{array}$} & \multirow{2}{*}{$\begin{array}{l}\text { Vaccinated } \\
\%(95 \% \mathrm{CI})\end{array}$} & \multirow{2}{*}{$\begin{array}{l}\begin{array}{l}\text { Strongly } \\
\text { hesitant }^{\mathrm{a}}\end{array} \\
\%(95 \% \mathrm{CI})\end{array}$} & \multicolumn{3}{|l|}{ Hesitant $^{\mathrm{a}}$} \\
\hline & & & & & $\%(95 \% \mathrm{CI})$ & $\mathrm{RR}(95 \% \mathrm{CI})$ & $\operatorname{aRR}(95 \% \mathrm{CI})$ \\
\hline \multicolumn{8}{|l|}{ Healthcare practitioners and support } \\
\hline Pharmacist & 895 & $92.2(90.5,94.0)$ & $\begin{array}{l}91.9 \text { (89.8, } \\
94.0)\end{array}$ & $4.3(2.7,6.0)$ & $6.9(4.9,8.9)$ & Reference & Reference \\
\hline Registered nurse/nurse practioner & 9701 & $93.2(92.6,93.7)$ & $\begin{array}{l}86.7 \text { (85.9, } \\
87.5)\end{array}$ & $8.0(7.4,8.7)$ & $\begin{array}{l}11.6(10.8, \\
12.3)\end{array}$ & $\begin{array}{l}1.68(1.19, \\
2.18)\end{array}$ & $\begin{array}{l}2.06(1.45, \\
2.67)\end{array}$ \\
\hline Therapist $^{\mathrm{b}}$ & 2438 & $94.5(93.5,95.5)$ & $\begin{array}{l}86.6(85.0 \\
88.3)\end{array}$ & $8.2(6.9,9.5)$ & $\begin{array}{l}12.1(10.5, \\
13.7)\end{array}$ & $\begin{array}{l}1.76(1.20 \\
2.31)\end{array}$ & $\begin{array}{l}2.08(1.42, \\
2.74)\end{array}$ \\
\hline Veterinarian & 647 & $97.6(96.4,98.7)$ & $\begin{array}{l}83.2(79.6, \\
86.9)\end{array}$ & $9.2(6.4,12.1)$ & $12.8(9.6,16.0)$ & $\begin{array}{l}1.86(1.15, \\
2.57)\end{array}$ & $\begin{array}{l}1.79(1.11, \\
2.47)\end{array}$ \\
\hline Physician or surgeon & 1847 & $94.1(92.9,95.4)$ & $\begin{array}{l}85.5(83.5 \\
87.6)\end{array}$ & $12.6(10.7,14.6)$ & $\begin{array}{l}13.9(11.9, \\
15.9)\end{array}$ & $\begin{array}{l}2.02(1.37 \\
2.67)\end{array}$ & $\begin{array}{l}2.28(1.55 \\
3.00)\end{array}$ \\
\hline Health technologist or technician & 4693 & $94.8(94.1,95.5)$ & $\begin{array}{l}82.9(81.6, \\
84.2)\end{array}$ & $9.4(8.4,10.4)$ & $\begin{array}{l}14.1 \text { (12.9, } \\
15.3)\end{array}$ & $\begin{array}{l}2.05(1.44, \\
2.67)\end{array}$ & $\begin{array}{l}1.87(1.30 \\
2.43)\end{array}$ \\
\hline Physician assistant & 656 & $94.6(92.5,96.6)$ & $\begin{array}{l}81.8(78.2, \\
85.5)\end{array}$ & $12.6(9.4,15.8)$ & $\begin{array}{l}15.8(12.3 \\
19.4)\end{array}$ & $\begin{array}{l}2.30(1.47, \\
3.14)\end{array}$ & $\begin{array}{l}2.48(1.60 \\
3.37)\end{array}$ \\
\hline Dentist & 480 & $94.8(92.4,97.1)$ & $\begin{array}{l}80.9 \text { (76.6, } \\
85.1)\end{array}$ & $13.7(9.8,17.5)$ & $\begin{array}{l}17.0(12.9, \\
21.1)\end{array}$ & $\begin{array}{l}2.47 \text { (1.54, } \\
3.39)\end{array}$ & $\begin{array}{l}2.51(1.61, \\
3.41)\end{array}$ \\
\hline Medical assistant & 1067 & $92.3(90.3,94.3)$ & $\begin{array}{l}78.6 \text { (75.4, } \\
81.8)\end{array}$ & $11.6(9.0,14.3)$ & $\begin{array}{l}17.4(14.5, \\
20.4)\end{array}$ & $\begin{array}{l}2.53(1.69, \\
3.38)\end{array}$ & $\begin{array}{l}2.22(1.49, \\
2.95)\end{array}$ \\
\hline Home health or personal care aide & 3349 & $83.7(82.2,85.1)$ & $\begin{array}{l}75.5(73.6, \\
77.4)\end{array}$ & $11.6(10.1,13.2)$ & $\begin{array}{l}18.0(16.2, \\
19.7)\end{array}$ & $\begin{array}{l}2.61(1.82, \\
3.40)\end{array}$ & $\begin{array}{l}2.21(1.53, \\
2.88)\end{array}$ \\
\hline $\begin{array}{l}\text { Licensed practical or licensed vocational } \\
\text { nurse }\end{array}$ & 2180 & $94.7(93.6,95.7)$ & $\begin{array}{l}77.4(75.3 \\
79.6)\end{array}$ & $13.3(11.6,15.0)$ & $\begin{array}{l}18.9(16.9 \\
20.8)\end{array}$ & $\begin{array}{l}2.74(1.90, \\
3.58)\end{array}$ & $\begin{array}{l}2.38(1.65 \\
3.11)\end{array}$ \\
\hline Nursing assistant or psychiatric aide & 1482 & $95.7(94.5,96.9)$ & $\begin{array}{l}74.4 \text { (71.7, } \\
77.1)\end{array}$ & $12.0(10.1,13.9)$ & $\begin{array}{l}19.5(17.2, \\
21.9)\end{array}$ & $\begin{array}{l}2.84 \text { (1.95, } \\
3.72)\end{array}$ & $\begin{array}{l}2.19(1.50, \\
2.87)\end{array}$ \\
\hline Emergency medical technicians/paramedics & 1073 & $97.0(95.8,98.1)$ & $\begin{array}{l}72.2 \text { (68.7, } \\
75.7)\end{array}$ & $20.1(16.9,23.3)$ & $\begin{array}{l}25.3(21.8, \\
28.7)\end{array}$ & $\begin{array}{l}3.67 \text { (2.50, } \\
4.84)\end{array}$ & $\begin{array}{l}2.69 \text { (1.83, } \\
3.54)\end{array}$ \\
\hline Other healthcare support & 11,568 & $73.9(73.0,74.8)$ & $\begin{array}{l}84.6(83.8, \\
85.4)\end{array}$ & $8.2(7.6,8.9)$ & $\begin{array}{l}12.4(11.6, \\
13.2)\end{array}$ & $\begin{array}{l}1.80(1.27 \\
2.33)\end{array}$ & $\begin{array}{l}1.79(1.26 \\
2.32)\end{array}$ \\
\hline Other healthcare practitioner & 1654 & $92.9(91.5,94.3)$ & $\begin{array}{l}78.6(76.2, \\
81.1) \\
\end{array}$ & $14.9(12.7,17.2)$ & $\begin{array}{l}20.0(17.6, \\
22.5)\end{array}$ & $\begin{array}{l}2.91(2.00, \\
3.82)\end{array}$ & $\begin{array}{l}3.17 \text { (2.19, } \\
4.16)\end{array}$ \\
\hline \multicolumn{8}{|l|}{ Educators } \\
\hline Postsecondary teacher & 4826 & $54.2(52.5,55.9)$ & $\begin{array}{l}94.9 \text { (94.2, } \\
95.7)\end{array}$ & $2.5(1.9,3.0)$ & $3.6(2.9,4.2)$ & Reference & Reference \\
\hline Secondary school teacher & 4837 & $91.9(91.1,92.8)$ & $\begin{array}{l}90.3(89.2, \\
91.3)\end{array}$ & $5.8(5.0,6.6)$ & $8.6(7.6,9.5)$ & $\begin{array}{l}2.40(1.89 \\
2.91)\end{array}$ & $\begin{array}{l}2.82(2.22, \\
3.43)\end{array}$ \\
\hline Elementary or middle school teacher & 6712 & $90.2(89.4,91.1)$ & $\begin{array}{l}88.9 \text { (88.0, } \\
89.8)\end{array}$ & $6.3(5.6,7.0)$ & $9.6(8.7,10.5)$ & $\begin{array}{l}2.70(2.16, \\
3.23)\end{array}$ & $\begin{array}{l}3.32(2.65, \\
4.00)\end{array}$ \\
\hline Preschool or kindergarten teacher & 3112 & $92.6(91.5,93.7)$ & $\begin{array}{l}81.7(80.0 \\
83.3)\end{array}$ & $9.0(7.8,10.3)$ & $\begin{array}{l}14.8(13.2, \\
16.3)\end{array}$ & $\begin{array}{l}4.15(3.29, \\
5.01)\end{array}$ & $\begin{array}{l}3.94(3.12, \\
4.77)\end{array}$ \\
\hline Other educator ${ }^{c}$ & 12,002 & $73.9(72.8,75.0)$ & $\begin{array}{l}87.7 \text { (87.0, } \\
88.5)\end{array}$ & $5.5(5.0,6.0)$ & $9.0(8.4,9.6)$ & $\begin{array}{l}2.53(2.04, \\
3.01)\end{array}$ & $\begin{array}{l}2.59(2.08, \\
3.09)\end{array}$ \\
\hline
\end{tabular}

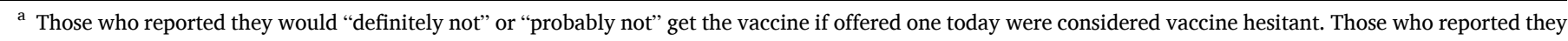
would "definitely not" were considered strongly hesitant.

b Including occupational, physical, respiratory, speech.

c Including teaching assistant, librarian, curator, or other.

entertainment/sports/media, and office/administrative support. Those in life/physical/social science, and education/training/library occupations led vaccine uptake, which was lowest among those in construction/extraction, installation/maintenance/repair, and farming/fishing/ forestry occupations.

Vaccine hesitancy varied widely by occupation category, with a prevalence of $<10 \%$ in computer/mathematical, life/physical/social science, education/training/library, and arts/design/entertainment/ sports/media, to $25-45 \%$ in construction/extraction, installation/ maintenance/repair, farming/fishing/forestry, protective services, and transportation/material moving, the military, and production, which includes food processing and meat packing (Table 1). Compared to educators, those in construction/extraction had 5 times the chance of vaccine hesitancy ( $R R=5.05$ [95\%CI 4.77-5.33]); with adjustment for demographics, including education, they still had $>3$-fold increased chance $(\mathrm{aRR}=3.29$ [3.10-3.47]).

COVID-19 vaccine hesitancy was similar among health care support
(14.4\% [95\%CI, 14.0-15.0]) and healthcare practitioners (14.5\%, [95\% CI, 14.0-15.0]). However, hesitancy rates varied by healthcare profession, ranging from $6.9 \%(95 \% \mathrm{CI}, 4.9-8.8)$ among pharmacists to $25.2 \%$ (95\%CI, 21.8-28.6) among emergency medical technicians/paramedics (Table 2). Registered nurses and nurse practitioners had relatively low hesitancy (11.6\% [10.8-12.3]), while nursing assistant and psychiatric technicians, professions with high patient contact, had a hesitancy prevalence of $18.8 \%$ (95\% CI, 16.9-20.8); differences in hesitancy between healthcare professions were attenuated with control for demographics. Vaccine hesitancy also varied by professions within education/training/library, with a range of $3.6 \%$ (95\%CI, 2.9-4.2) among post-secondary teachers to $14.8 \%$ (13.2-16.3) among preschool/ kindergarten teachers.

Reasons for vaccine hesitancy among all employed respondents, and specifically among healthcare workers and educators are reported in Table 3. Over half of employed hesitant participants reported concern about side effects (51.7\%, 95\%CI, 51.1-52.2) and not trusting COVID-19 
Table 3

Prevalence of reasons for vaccine hesitancy among hesitant ${ }^{\mathrm{a}}$ employed 18-64 year-old US adults overall, and for health care practitioners, healthcare support, and educators, April 20-May 19, 2021.

\begin{tabular}{|c|c|c|c|c|}
\hline & $\begin{array}{l}\text { Total } \\
\text { employed } \\
\mathrm{N}=55375\end{array}$ & $\begin{array}{l}\mathrm{HC} \\
\text { Practitioners/ } \\
\text { Technicians } \\
\mathrm{N}=3602\end{array}$ & $\begin{array}{l}\mathrm{HC} \\
\text { Support } \\
\mathrm{N}=2447\end{array}$ & $\begin{array}{l}\text { Educators } \\
\mathrm{N}=2580\end{array}$ \\
\hline & \multicolumn{4}{|c|}{$\%(95 \% \mathrm{CI})$} \\
\hline \multicolumn{5}{|l|}{ Reasons } \\
\hline Side effects & $\begin{array}{l}51.7(51.1, \\
52.2)\end{array}$ & $52.5(50.5,54.4)$ & $\begin{array}{l}54.6 \\
(52.1 \\
57.1)\end{array}$ & $\begin{array}{l}56.3(54.0, \\
58.6)\end{array}$ \\
\hline $\begin{array}{l}\text { Don't trust COVID- } \\
19 \text { vaccine }\end{array}$ & $\begin{array}{l}51.3(50.8, \\
51.8)\end{array}$ & $48.0(46.0,50.0)$ & $\begin{array}{l}48.9 \\
(46.5 \\
51.4)\end{array}$ & $\begin{array}{l}44.9 \text { (42.7, } \\
47.2)\end{array}$ \\
\hline Do not need & $\begin{array}{l}45.1(44.6, \\
45.7)\end{array}$ & $40.3(38.3,42.3)$ & $\begin{array}{l}36.4 \\
(34.0 \\
38.8)\end{array}$ & $\begin{array}{l}41.7(39.4, \\
44.0)\end{array}$ \\
\hline $\begin{array}{l}\text { Don't trust } \\
\text { government }\end{array}$ & $\begin{array}{l}44.6(44.1, \\
45.2)\end{array}$ & $35.8(33.8,37.7)$ & $\begin{array}{l}38.3 \\
(35.8 \\
40.7)\end{array}$ & $\begin{array}{l}34.5(32.3, \\
36.8)\end{array}$ \\
\hline $\begin{array}{l}\text { Wait to see if safe } \\
\text { then maybe later }\end{array}$ & $\begin{array}{l}35.2(34.7, \\
35.8)\end{array}$ & $39.2(37.2,41.1)$ & $\begin{array}{l}42.4 \\
(39.9 \\
44.9)\end{array}$ & $\begin{array}{l}43.8(41.5, \\
46.1)\end{array}$ \\
\hline $\begin{array}{l}\text { Don't know if it will } \\
\text { work }\end{array}$ & $\begin{array}{l}24.2(23.8, \\
24.7)\end{array}$ & $22.2(20.5,23.9)$ & $\begin{array}{l}24.3 \\
(22.2 \\
26.4)\end{array}$ & $\begin{array}{l}24.3(22.3, \\
26.3)\end{array}$ \\
\hline Allergic reaction & $\begin{array}{l}22.6(22.1, \\
23.0)\end{array}$ & $22.0(20.5,23.6)$ & $\begin{array}{l}28.2 \\
(25.9 \\
30.4)\end{array}$ & $\begin{array}{l}24.9 \text { (22.9, } \\
26.9)\end{array}$ \\
\hline Don't like vaccines & $\begin{array}{l}15.0(14.6, \\
15.4)\end{array}$ & $10.8(9.6,12.1)$ & $\begin{array}{l}12.9 \\
(10.8 \\
15.0)\end{array}$ & $\begin{array}{l}13.1(11.4, \\
14.8)\end{array}$ \\
\hline $\begin{array}{l}\text { Other people need } \\
\text { more }\end{array}$ & $\begin{array}{l}14.2(13.8, \\
14.6)\end{array}$ & $11.9(10.6,13.2)$ & $\begin{array}{l}12.7 \\
(11.2 \\
14.3)\end{array}$ & $\begin{array}{l}14.2(12.5, \\
15.8)\end{array}$ \\
\hline $\begin{array}{l}\text { Doctor not } \\
\text { recommended }\end{array}$ & $\begin{array}{l}9.4(9.0 \\
9.7)\end{array}$ & $9.3(8.1,10.4)$ & $\begin{array}{l}7.7(6.5, \\
8.9)\end{array}$ & $\begin{array}{l}10.8(9.4, \\
12.2)\end{array}$ \\
\hline $\begin{array}{l}\text { Safety concern } \\
\text { because of health } \\
\text { condition }\end{array}$ & $\begin{array}{l}9.0(8.7, \\
9.3)\end{array}$ & $11.9(10.7,13.2)$ & $\begin{array}{l}13.3 \\
(11.8 \\
14.9)\end{array}$ & $\begin{array}{l}14.8(13.1, \\
16.5)\end{array}$ \\
\hline Against religion & $\begin{array}{l}8.6(8.3, \\
8.9)\end{array}$ & $8.7(7.6,9.8)$ & $\begin{array}{l}8.3(6.5 \\
10.1)\end{array}$ & $\begin{array}{l}8.6(7.1, \\
10.0)\end{array}$ \\
\hline $\begin{array}{l}\text { Currently/planning } \\
\text { to be pregnant/ } \\
\text { breastfeeding }\end{array}$ & $\begin{array}{l}6.9(6.6 \\
7.1)\end{array}$ & $14.3(12.9,15.7)$ & $\begin{array}{l}12.0 \\
(10.3 \\
13.6)\end{array}$ & $\begin{array}{l}13.9(12.2, \\
15.5)\end{array}$ \\
\hline Cost & $\begin{array}{l}3.3(3.1 \\
3.5)\end{array}$ & $2.1(1.5,2.7)$ & $\begin{array}{l}2.4(1.7 \\
3.1)\end{array}$ & $\begin{array}{l}1.9(1.3, \\
2.5)\end{array}$ \\
\hline Other & $\begin{array}{l}17.1(16.7, \\
17.5)\end{array}$ & $15.9(14.5,17.4)$ & $\begin{array}{l}12.9 \\
(11.3 \\
14.5)\end{array}$ & $\begin{array}{l}13.4(11.8, \\
15.0)\end{array}$ \\
\hline
\end{tabular}

$\mathrm{HC}=$ healthcare.

a Those who reported they would "definitely not" or "probably not" get the vaccine if offered one today were considered vaccine hesitant.

vaccines $(51.3 \%, 95 \% \mathrm{CI}, 50.8-51.8)$, whereas only $15.0 \%$ (95\%CI, 14.6-15.4) didn't like vaccines in general. Other reasons endorsed by over one-third of respondents were: didn't believe they needed the vaccine, didn't trust the government, were waiting to see if the vaccine was safe. The prevalence of reasons for COVID-19 vaccine hesitancy among healthcare practitioners/technicians, healthcare support and educators mostly mirrored that of the overall workforce; however, for all three groups, not trusting the government was a less common reason, while waiting to see if safe, and currently or planning to be pregnant or breastfeeding were more common (Table 3 ).

Reasons for vaccine hesitancy among the five occupations with the highest prevalence of hesitancy are reported in Table 4. Compared to all employed hesitant participants, a higher percentage of respondents with jobs in construction/extraction, installation/maintenance/repair, farming/fishing/forestry, protective services, or transportation/material moving reported distrust of the government and not needing the vaccine. With the exception of farming/forestry/fishing, these occupations were also more likely to not trust the vaccine. In contrast, a smaller percentage of those in construction/extraction, and farming/ fishing/forestry, reported worry about side effects, an allergic reaction and waiting to see if the vaccine was safe (Table 4).

Reasons for hesitancy among 5 additional occupations selected due to high-density indoor workspace or significant client contact (military, production, food preparation/serving, personal care/service, community and social service) are provided in eTable4. Compared to all employed hesitant participants, a higher percentage of respondents in the military reported distrust in the COVID-19 vaccine, disbelief of need and waiting to see if safe; a higher percentage of those in production reported distrust of the government; a higher percentage of those in community and social service or personal care/service reported waiting to see if safe, safety concern because of health conditions and currently/ planning to be pregnant or breastfeeding; in addition, among those in personal care/service, concern regarding side effects or an allergic reaction and against religion were more common; finally, a higher percentage of those in food preparation/serving reported concerns regarding side effects or an allergic reaction, waiting to see if safe, other people need more than me, and currently/planning to be pregnant or breastfeeding.

\section{Discussion}

In this massive national survey of adults 18-64 years, COVID-19 vaccine hesitancy decreased by just over one-third from January to May 2021. While this is a promising finding, $19 \%$ of the workforce, and $22 \%$ of adults working outside the home in May reported vaccine hesitancy. Furthermore, there was a large disparity in vaccine hesitancy by occupation, with a five-fold difference between the lowest and highest values. While adjustment for demographics reduced the differences in hesitancy between occupation, one-third of occupation categories still had a 2-3.3 fold higher hesitancy that the lowest hesitancy occupations with adjustment. With the emergence of more infectious COVID variants (CDC, 2019), addressing COVID-19 vaccine hesitancy to improve vaccine uptake is a priority for pandemic control, particularly among the workforce.

Occupation categories with the highest hesitancy (construction/ extraction, installation/maintenance/repair, farming/fishing/forestry, protective service, and transportation/material moving), include some that have suffered workplace outbreaks, such as agriculture and protective service (Althouse et al., 2020; Graves et al., 2014; Waltenburg et al., 2021). The majority of hesitant participants in these occupations had strong hesitancy (i.e., responded "definitely not") and reported not trusting the government and/or the COVID-19 vaccine, indicating that their hesitancy may be based in strong beliefs about the government or the vaccine development process. Further, they were more likely than all employed hesitant participants to believe they do not need the vaccine. In some of these professions, individuals may work primarily outside or in uncrowded conditions and feel less at risk of contracting COVID-19. Thus, their reasons for hesitancy indicate a need for public health campaigns to increase trust in the COVID-19 vaccine and the government, and to increase awareness of the benefits of a COVID-19 vaccine to employees and their community in order to address the belief that some individuals do not need the vaccine.

Given the variation in hesitancy by occupational groups, public health and medical workers could seek to understand and address reasons for hesitancy in specific workplace communities by building partnerships in occupations with high vaccine hesitancy. Workplace vaccination clinics have the potential to address several potential barriers to COVID-19 vaccination, e.g., difficulty scheduling, transportation, travel and time requirements, including unpaid time off of work, and of going to an unfamiliar location (Luthy et al., 2016; CDC; CDC, 2021). In addition to clinics, employers can promote vaccine access by ensuring paid time off and offering transportation to workers to receive vaccines offsite. Workplace efforts can address poor 
Table 4

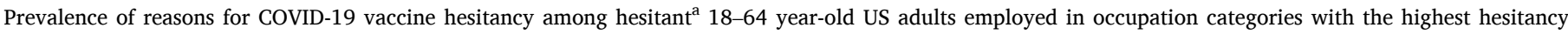
prevalence.

\begin{tabular}{|c|c|c|c|c|c|}
\hline & $\begin{array}{l}\text { Construction and } \\
\text { extraction }^{\mathrm{a}} \mathrm{N}=2470\end{array}$ & $\begin{array}{l}\text { Installation, maintenance, } \\
\text { repair } \mathrm{N}=3030\end{array}$ & $\begin{array}{l}\text { Farming, fishing, and } \\
\text { forestry } N=770\end{array}$ & $\begin{array}{l}\text { Protective service } \\
\mathrm{N}=1154\end{array}$ & $\begin{array}{l}\text { Transportation and material } \\
\text { moving }^{\mathrm{b}} \mathrm{N}=3421\end{array}$ \\
\hline & \multicolumn{5}{|c|}{$\%(95 \% \mathrm{CI})$} \\
\hline \multicolumn{6}{|l|}{ Reasons } \\
\hline Side effects & $43.2(40.8,45.7)$ & $50.4(48.2,52.6)$ & $44.1(39.7,48.5)$ & $52.2(48.8,55.6)$ & $49.6(47.6,51.6)$ \\
\hline Don't trust COVID-19 vaccine & $56.6(54.2,59.0)$ & $55.4(53.2,57.6)$ & $52.9(48.5,57.2)$ & $57.9(54.6,61.2)$ & $56.2(54.2,58.2)$ \\
\hline Do not need & $53.6(51.1,56.1)$ & $56.1(53.9,58.3)$ & $53.0(48.6,57.4)$ & $53.1(49.7,56.4)$ & $49.3(47.3,51.3)$ \\
\hline Don't trust government & $54.8(52.3,57.3)$ & $53.9(51.7,56.2)$ & $52.3(48.0,56.7)$ & $50.7(47.4,54.1)$ & $51.7(49.7,53.8)$ \\
\hline $\begin{array}{l}\text { Wait to see if safe then maybe } \\
\text { later }\end{array}$ & $24.0(21.9,26.1)$ & $29.3(27.3,31.4)$ & $23.0(19.0,26.9)$ & $32.8(29.6,36.1)$ & $31.0(29.1,32.9)$ \\
\hline Don't know if it will work & $24.7(22.5,26.9)$ & $24.8(22.9,26.6)$ & $21.4(17.9,25.0)$ & $29.0(25.9,32.1)$ & $25.3(23.5,27.1)$ \\
\hline Allergic reaction & $17.0(15.2,18.9)$ & $19.4(17.7,21.2)$ & $15.1(12.3,18.0)$ & $20.7(18.1,23.4)$ & $21.3(19.8,22.9)$ \\
\hline Don't like vaccines & $15.6(13.9,17.3)$ & $16.9(15.3,18.6)$ & $15.8(12.5,19.1)$ & $13.7(11.5,16.0)$ & $17.3(15.7,18.9)$ \\
\hline Other people need more & $12.1(10.4,13.8)$ & $14.8(13.2,16.5)$ & $10.7(7.9,13.5)$ & $15.0(12.5,17.4)$ & $14.2(12.6,15.7)$ \\
\hline Doctor not recommended & $8.8(7.4,10.3)$ & $10.0(8.7,11.3)$ & $7.6(5.6,9.6)$ & $11.3(9.1,13.4)$ & $9.1(8.0,10.3)$ \\
\hline $\begin{array}{l}\text { Safety concern because of } \\
\text { health condition }\end{array}$ & $4.4(3.5,5.2)$ & $5.5(4.5,6.4)$ & $6.1(4.0,8.1)$ & $6.4(4.9,7.8)$ & $6.6(5.7,7.4)$ \\
\hline Against religion & $8.1(6.9,9.4)$ & $8.4(7.2,9.6)$ & $10.4(7.8,13.0)$ & $8.9(7.0,10.7)$ & $10.0(8.8,11.2)$ \\
\hline $\begin{array}{l}\text { Currently/planning to be } \\
\text { pregnant/breastfeeding }\end{array}$ & $2.1(1.4,2.8)$ & $1.8(1.2,2.4)$ & $4.6(2.9,6.4)$ & $3.8(2.5,5.1)$ & $2.4(1.8,3.1)$ \\
\hline Cost & $3.5(2.5,4.4)$ & $3.1(2.3,3.8)$ & $2.4(1.2,3.6)$ & $3.5(2.1,4.8)$ & $4.1(3.2,4.9)$ \\
\hline Other & $19.9(17.9,21.9)$ & $18.9(17.2,20.5)$ & $19.5(16.2,22.7)$ & $18.0(15.5,20.6)$ & $17.6(16.0,19.2)$ \\
\hline
\end{tabular}

aThose who reported they would "definitely not" or "probably not" get the vaccine if offered one today were considered vaccine hesitant.

${ }^{a}$ Including oil, gas, mining, or quarrying.

bIncluding delivery services.

understanding of the risks and benefits, and lack of vaccination being the norm, by providing population-specific educational messaging and positive peer pressure (Luthy et al., 2016; CDC; CDC, 2021). The Centers for Disease Control and Prevention (CDC) provides COVID-19 vaccination audience-specific toolkits to promote vaccine acceptance, including an essential workers toolkit (Promoting Vaccination in the Workplace, 2021), and guidance to employers on hosting workplace vaccination clinics (Promoting Vaccination in the Workplace, 2021). They advise including management, human resources, employees and labor representatives, as appropriate, in the planning process, and using multiple strategies to promote and encourage participation in the vaccination clinics, e.g., encouraging managers and leaders to get vaccinated first. Just as celebrities have promoted vaccination to the public and Black health care workers have had success engaging Black communities (Lopez-Villafana, 2021), workplace-focused campaigns could feature prominent and ordinary figures from specific workplaces or occupations discussing why they got vaccinated (Burden et al., 2021).

Among healthcare workers, several professions with high patient contact (e.g., nursing assistants/psychiatric aides) reported hesitancy > $15 \%$. This is concerning as patients are often at higher risk of hospitalization or death from COVID-19 than the general population, based on their age or health status. Published guidance on promoting vaccinations among healthcare workers (Yue et al., 2019; CDC, 2014) may serve as a starting point for COVID-19 specific efforts.

Hesitancy among educators was generally low. However, $15 \%$ of preschool and kindergarten teachers and $10 \%$ of elementary school teachers, who teach children not yet eligible for a COVID-19 vaccine, were hesitant. While some universities and private schools are requiring students, staff and faculty to be vaccinated before the start of the 2021 fall semester (Anderson et al., 2021; Nietzel et al., 2021), most private and public preschool and elementary schools have no vaccine mandates (Howard, 2021). Many also lack masking mandates (Chuck, 2021; King et al., 2021), making vaccination even more important.

A striking finding was that participants working outside the home reported COVID-19 vaccine hesitancy at more than twice the rate of those working from home. This may reflect the observed difference in hesitancy by occupation, as working from home was more common in some occupations than others. This finding may also reflect that those who are more worried about COVID-19, who as a group have less
COVID-19 vaccine hesitancy (King et al., 2021), are choosing to work from home when possible.

\subsection{Study limitations and strengths}

Cross-sectional samples were used to evaluate time trends, and the sample representativeness may have been affected by the recruitment method and low response rate. Specifically, this study used a novel sampling method with a soft ask. Responses were weighted to match the age, gender, and state profile of the US population (Barkay et al., 2020), but representativeness within each occupational category is not guaranteed. Additionally, studies from the previous decade found differences in personality traits between Facebook users and non-users (Ljepava et al., 2013; Ryan and Xenos, 2011). While we do not expect those exact findings to hold a decade later in the much larger and more diverse Facebook user population (Auxier and Anderson, 2021), the Facebook user and general US populations are expected to differ, and we could not control for unmeasured differences between them or the impact of receiving vaccine-related content through Facebook itself. Compared to the American Community Survey 2015-2019 5-year Data Release (US Census Bureau. American Community Survey 5-Year Data, 2021), demographics of the weighted sample are similar to the US population, but white race and higher education are slightly over-represented, and vaccine uptake is over-represented (Daly et al., 2021). Thus, overall hesitancy prevalence estimates were likely underestimated. A study strength is that vaccinated individuals were included in the vaccine accepting (i.e., not hesitant) group, as access to vaccination varied by occupational group over the time studied. Thus, assessment of time trends or comparisons between occupation categories should be valid.

Additional study strengths include the timing of our study (i.e., during the first five months of the COVID-19 vaccine rollout) and our large geographically and occupationally diverse sample, which allowed for comparisons by month and occupation. This large-scale national sample with detailed data on occupational categories and respondent characteristics is, to the author's knowledge, the best US data available on COVID-19 hesitancy by employment and occupation. 


\section{Conclusions}

Vaccine hesitancy among US adults 18-64 years decreased in the first five months of the US COVID-19 vaccine rollout. However, with approximately one in five members of the US workforce hesitant in May 2021, and some occupational categories reporting hesitancy at twice this rate, vaccine hesitancy remains a threat to COVID-19 pandemic control. This report identified occupations with high rates of COVID-19 vaccine hesitancy in the workforce and in specific professions to help public health and health care workers target interventions and address specific concerns to increase vaccination rates, potentially via workplace-focused campaigns and onsite vaccination clinics. Messaging about safety, addressing trust, and clarifying the value of vaccinations to prevent COVID-19 is needed.

\section{CRediT authorship contribution statement}

Wendy C. King: Conceptualization, Investigation, Methodology, Visualization, Writing - original draft, Writing - review \& editing. Max Rubinstein: Data curation, Formal analysis, Investigation, Methodology, Software, Validation, Visualization, Writing - review \& editing. Alex Reinhart: Conceptualization, Data curation, Formal analysis, Funding acquisition, Investigation, Methodology, Project administration, Resources, Software, Supervision, Validation, Visualization, Writing - review \& editing. Robin J. Mejia: Conceptualization, Investigation, Methodology, Resources, Supervision, Visualization, Writing original draft, Writing - review \& editing.

\section{Acknowledgements}

The authors would like to thank the Delphi group at Carnegie Mellon University for input and support on the survey instrument. Wichada La Motte-Kerr, MPH, of Delphi contributed to the development and deployment of the survey and received compensation for her contributions to the study. We thank Paul Henneberger, ScD and Jean CoxGanser, PhD of NIOSH for developing occupation items used in the survey, and we thank Sarah LaRocca, PhD and Katherine Morris, $\mathrm{PhD}$ of Facebook for contributions to the survey instrument design.

\section{Declaration of Competing Interest}

Drs. King, Mejia and Mr. Rubinstein have no conflict of interest to report. Dr. Reinhart received salary support from an unrestricted gift from Facebook.

\section{Funding/Support}

This material is based upon work supported by Facebook (unrestricted gift) and a cooperative agreement from the Centers for Disease Control and Prevention (U01IP001121).

\section{Role of the Funder}

Facebook was involved in the design and conduct of the study. The CDC provided funding only. Neither Facebook nor the Centers for Disease Control and Prevention had a role in the collection, management, analysis, and interpretation of the data; preparation, review, or approval of the manuscript; or decision to submit the manuscript for publication. Any opinions, findings and conclusions or recommendations expressed in this material are those of the authors and do not necessarily reflect the views of Facebook or the Centers for Disease Control and Prevention.

\section{Appendix A. Supplementary data}

Supplementary data to this article can be found online at https://doi. org/10.1016/j.pmedr.2021.101569.

\section{References}

Althouse, B.M., Wenger, E.A., Miller, J.C., Scarpino, S.V., Allard, A., Hébert-Dufresne, L., $\mathrm{Hu}, \mathrm{H} ., 2020$. Superspreading events in the transmission dynamics of SARS-CoV-2: opportunities for interventions and control. PLoS Biol. 18 (11), e3000897. https:// doi.org/10.1371/journal.pbio.3000897.

American Community Survey. Census - Table Results. Accessed August 2, 2021. https ://data.census.gov/cedsci/table?d=ACS\%201-Year\%20Estimates\%20Data\%20Profi les\&tid=ACSDP1Y2019.DP05.

Anderson, N., Svrluga, S., Stanley-Becker, I., Lumpkin, L., Aguilar, M. 2021. Colleges want students to get a coronavirus vaccine. But they're split on requiring the shots. Washington Post. https://www.washingtonpost.com/education/2021/06/23/colle ges-covid-vaccine-mandates-divide/. Published June 23. Accessed August 10, 2021.

Auxier, B., Anderson, M. 2021. Social Media Use in 2021. Pew Research Center: Internet, Science \& Tech. Published April 7, 2021. Accessed August 12, 2021. https://www.pe wresearch.org/internet/2021/04/07/social-media-use-in-2021/.

Baack, B.N. COVID-19 Vaccination Coverage and Intent Among Adults Aged 18-39 Years - United States, March-May 2021. MMWR Morb. Mortal Wkly. Rep. 2021, 70. doi: 10.15585/mmwr.mm7025e2.

Barkay, N., Cobb, C., Eilat, R., et al. Weights and Methodology Brief for the COVID-19 Symptom Survey by University of Maryland and Carnegie Mellon University, in Partnership with Facebook. arXiv:200914675 [cs]. Published online October 6, 2020. Accessed May 26, 2021. http://arxiv.org/abs/2009.14675.

Burden, S., Henshall, C., Oshikanlu, R., 2021. Harnessing the nursing contribution to COVID-19 mass vaccination programmes: Addressing hesitancy and promoting confidence. J. Adv. Nurs. 77 (8), e16-e20. https://doi.org/10.1111/jan.14854.

CDC. Worksite Health: Seasonal Influenza Vaccinations Using Interventions with On-Site, Free, Actively Promoted Vaccinations - Healthcare Workers. The Guide to Community Preventive Services (The Community Guide). Published April 17, 2014. Accessed August 10, 2021. https://www.thecommunityguide.org/findings/worksit e-health-seasonal-influenza-vaccinations-healthcare-on-site.

CDC. Coronavirus Disease 2019 (COVID-19). Centers for Disease Control and Prevention. Published February 11, 20Accessed August 10, 2021. https://www.cdc.gov/coro navirus/2019-ncov/variants/delta-variant.html.

Centers for Disease Control and Prevention. Data Access - Urban Rural Classification Scheme for Counties. Published December 2, 2019. Accessed June 25, 2021. htt ps://www.cdc.gov/nchs/data_access/urban_rural.htm.

CDC. Interventions to Promote Seasonal Influenza Vaccinations among Non-Healthcare Workers Interventions with On-site, Reduced Cost, Actively Promoted Vaccinations Task Force Finding and Rationale Statement. Centers for Disease Control.:2.

CDC, CDC. COVID-19 Vaccine Communication Toolkit for Essential Workers. Centers for Disease Control and Prevention. Published July 29, 2021. Accessed August 10, 2021. https://www.cdc.gov/coronavirus/2019-ncov/vaccines/toolkits/essential-workers. html.

Chuck, E. From lawsuits to sleepless nights, parents rattled by school mask policies. NBC News. Accessed August 10, 2021. https://www.nbcnews.com/news/us-news/many -classrooms-won-t-require-masks-children-year-it-s-n1274657.

Daly, M., Jones, A., Robinson, E., 2021. Public trust and willingness to vaccinate against COVID-19 in the US From October 14, 2020, to March 29, 2021. JAMA 325 (23), 2397. https://doi.org/10.1001/jama.2021.8246.

Freedman, David A., 2006. On the so-called "Huber sandwich estimator" and "robust standard errors". Am. Stat. 60 (4), 299-302.

Gold JAW. Clusters of SARS-CoV-2 Infection Among Elementary School Educators and Students in One School District - Georgia, December 2020-January 2021. MMWR Morb. Mortal Wkly. Rep. 2021;70. doi:10.15585/mmwr.mm7008e4.

Graves, M.A., Harris, J.R., Hannon, P.A., Hammerback, K., Ahmed, F., Zhou, C., 2014 Workplace-based influenza vaccination promotion practices among large employers in the United States. J. Occup. Environ. Med. 56 (4), 397-402. https://doi.org/ 10.1097/JOM.0000000000000115.

Howard, J. 2021. Some states move to block Covid-19 vaccine requirements in public schools - CNN. CNN. Accessed August 10. https://www.cnn.com/2021/07/13/heal th/covid-19-vaccine-requirements-schools-analysis-wellness/index.html.

Khubchandani, J., Sharma, S., Price, J.H., Wiblishauser, M.J., Sharma, M., Webb, F.J., 2021. COVID-19 vaccination hesitancy in the United States: a rapid national assessment. J Community Health. 46 (2), 270-277. https://doi.org/10.1007/ s10900-020-00958-x.

King, W.C., Rubinstein, M., Reinhart, A., Mejia, R.J. Time Trends and Factors Related to COVID-19 Vaccine Hesitancy from January-May 2021 among US Adults: Findings from a Large-Scale National Survey.; 2021:2021.07.20.21260795. doi:10.1101/ 2021.07.20.21260795.

Ljepava, N., Orr, R.R., Locke, S., Ross, C., 2013. Personality and social characteristics of Facebook non-users and frequent users. Comput. Human Behav. 29 (4), 1602-1607. https://doi.org/10.1016/j.chb.2013.01.026.

Lopez-Villafana A. Black nurses bring vaccine to community centers, churches to target under-served people. San Diego Union-Tribune. Published March 17, 2021. Accessed June 25, 2021. https://www.sandiegouniontribune.com/communities/san-diego/st ory/2021-03-17/san-diegos-black-nurses-focus-on-vaccinating-black-residents-in-s outheastern-san-diego.

Luthy, K.E., Bainum, J.L., Beckstrand, R.L., Macintosh, J.L.B., Eden, L.M., Saunders, B., 2016. Promoting adult pertussis vaccination in the workplace. Workplace Health Saf. 64 (6), 269-278. https://doi.org/10.1177/2165079916628883.

Malik, A.A., McFadden, S.M., Elharake, J., Omer, S.B., 2020. Determinants of COVID-19 vaccine acceptance in the US. EClinicalMedicine 26, 100495. https://doi.org/ 10.1016/j.eclinm.2020.100495.

Nietzel, M.T. 2021. In A Divided America, Every One Of Nation's Top 25 Universities Now Requiring Students To Be Vaccinated. Forbes. Accessed August 10, 2021. htt 
ps://www.forbes.com/sites/michaeltnietzel/2021/07/30/in-a-divided-america-ev ery-one-of-nations-top-25-universities-now-requiring-students-to-be-vaccinated/.

Pew Research Center. Growing Share of Americans Say They Plan To Get a COVID-19 Vaccine - or Already Have. Pew Research Center Science \& Society. Published March 5, 2021. Accessed August 2, 2021. https://www.pewresearch.org/science/2021/03 05/growing-share-of-americans-say-they-plan-to-get-a-covid-19-vaccine-or-alrea dy-have/.

Promoting Vaccination in the Workplace | CDC. Published May 6, 2021. Accessed August 10, 2021. https://www.cdc.gov/flu/business/promoting-vaccines-workplace.htm.

Razai, M.S., Chaudhry, U.A.R., Doerholt, K., Bauld, L., Majeed, A., 2021. Covid-19 vaccination hesitancy. BMJ 373, n1138. https://doi.org/10.1136/bmj.n1138.

Rosenthal, N., Cao, Z., Gundrum, J., Sianis, J., Safo, S., 2020. Risk factors associated with in-hospital mortality in a US National sample of patients with COVID-19. JAMA Netw. Open 3 (12), e2029058. https://doi.org/10.1001/ jamanetworkopen.2020.29058.

Ryan, T., Xenos, S., 2011. Who uses Facebook? An investigation into the relationship between the Big Five, shyness, narcissism, loneliness, and Facebook usage. Comput. Human Behav. 27 (5), 1658-1664. https://doi.org/10.1016/j.chb.2011.02.004.

Salomon, J.A., Reinhart, A., Bilinski, A., et al. The U.S. COVID-19 Trends and Impact Survey, 2020-2021: Continuous real-time measurement of COVID-19 symptoms, risks, protective behaviors, testing and vaccination. medRxiv. Published online July 26, 2021:2021.07.24.21261076. doi:10.1101/2021.07.24.21261076.

Schaffer DeRoo, S., Pudalov, N.J., Fu, L.Y., 2020. Planning for a COVID-19 vaccination program. JAMA 323 (24), 2458. https://doi.org/10.1001/jama.2020.8711.
Szilagyi, P.G., Thomas, K., Shah, M.D., Vizueta, N., Cui, Y., Vangala, S., Kapteyn, A., 2021. National trends in the US Public's likelihood of getting a COVID-19 Vaccine-April 1 to December 8, 2020. JAMA 325 (4), 396. https://doi.org/ 10.1001/jama.2020.26419.

Standard Occupational Classification (SOC) System. Accessed July 29, 2021. https ://www.bls.gov/soc/.

US Census Bureau. Race/Ethnicity and the 2020 Census. Census 20/20. Accessed August 2, 2021. https://www.census2020now.org/faces-blog/same-sex-households-202 0-census-r3976.

US Census Bureau. Educational Attainment. The United States Census Bureau. Accessed August 12, 2021. https://www.census.gov/topics/education/educational-attainme nt/about.html.

US Census Bureau. American Community Survey 5-Year Data (2009-2019). The United States Census Bureau. Accessed June 27, 2021. https://www.census.gov/data/deve lopers/data-sets/acs-5year.html.

Waltenburg, M.A., Rose, C.E., Victoroff, T., et al. Coronavirus Disease among Workers in Food Processing, Food Manufacturing, and Agriculture Workplaces - 27(1) January 2021 - Emerging Infectious Diseases journal - CDC. doi:10.3201/ eid2701.203821.

Yue, X., Black, C., Ball, S., Donahue, S., de Perio, M.A., Laney, A.S., Greby, S., 2019. Workplace interventions and vaccination-related attitudes associated with influenza vaccination coverage among healthcare personnel working in long-term care facilities, 2015-2016 Influenza season. J. Am. Med. Dir. Assoc. 20 (6), 718-724. https://doi.org/10.1016/j.jamda.2018.11.029. 